Génét. Sél. Evol., 1983, 15 (2), 251-256

\title{
NOTE \\ Taux de corticostérone plasmatique, variation de poids et de température corporelle de poules Leghorn blanches naines (dw) et normales (Dw+) en réponse à une privation d'aliment
}

\author{
P. ROMBAUTS *, A. BORDAS **, A.K. BANERJEE ** et P. MERAT ** \\ avec la collaboration technique de J. BerTin * et J. DurAo ** \\ I.N.R.A., * Station de Physiologie animale. \\ ** Laboratoire de Génétique factorielle, Centre de Recherches zootechniques, F 78350 Jouy-en-Josas
}

\begin{abstract}
Résumé
Des poules Leghorns naines (dw) et normales $\left(\mathrm{Dw}^{+}\right)$de même origine âgées d'environ 12 mois ont été soumises à la diète hydrique (privation de l'aliment seul) pendant 60 heures. Le poids corporel, la température rectale et le taux de corticostérone plasmatique ont été mesurés avant traitement et après un peu moins de 48 heures de jeûne. La baisse de poids en pourcentage du poids initial et la baisse de température rectale sont significativement plus importantes pour les poules dw. Le taux de corticostérone, déjà plus élevé au départ chez les naines, augmente davantage pour ce génotype après privation d'aliment. La reprise de poids dans le mois qui suit, en $\%$ du poids en fin de jeûne, semble par contre supérieure pour les poules $\mathrm{dw}$.
\end{abstract}

Mots clés : poule, nanisme, agression, jeûne, corticostérone

\section{Summary}

Plasma corticosterone, body weight and body temperature variation of dwarf $(d w)$ and normal $\left(D w^{+}\right)$White leghorn hens in response to starvation

Twelve month old Dwarf (dw) and normal $\left(\mathrm{Dw}^{+}\right)$white Leghorn hens of the same origin were deprived of food for 60 hours. Body weight, rectal temperature and plasma corticosterone level were measured before treatment and after almost 48 hours starvation. Both body weight loss, as a percentage of initial weight and decrease in rectal temperature were significantly higher for dw hens. Corticosterone level was higher for dwarf birds before starvation and increased more during the treatment. During the month following starvation, weight gains, expressed as a percentage of body weight at the end of starvation, seemed to be greater for dw hens.

Key words : fowl, dwarfism, stress, starvation, corticosterone. 


\section{Introduction}

Des différences génétiques dans le taux de corticostérone plasmatique, particulièrement en réponse à certaines agressions, ont été mises en évidence chez les volailles. FrEEMAN (1976) cite plusieurs résultats dans ce sens. Une sélection de lignées divergentes de ce point de vue a été réalisée (Gross et Siegel, 1973, 1981). MAULdin (1978), MAULdin et al. (1978) montrent l'effet du gène de nanisme lié au sexe sur ce paramètre chez des jeunes dans deux lignées différant par le poids corporel moyen. Ils concluent que l'effet de ce gène est influencé par le reste du génotype.

Le présent travail compare des poules naines $(\mathrm{dw})$ et normales $\left(\mathrm{Dw}^{+}\right)$en période de ponte, avant et après une " agression " constituée par une privation d'aliment. Ce facteur particulier a été choisi, du fait que sa présence ne peut être totalement exclue dans des formes d'élevage traditionnelles ou même semi-intensives.

\section{Matériel et méthodes}

La comparaison porte sur 55 poules, $28 \mathrm{Dw}^{+}$et $27 \mathrm{dw}$, sœurs ou demi-sœurs des premières. Ces poules, âgées d'environ 12 mois (octobre 1981) se trouvaient en cages individuelles. Elles avaient auparavant été contrôlées pour diverses performances de ponte et faisaient partie du lot témoin de l'expérience décrite par BANERJEE et al. (1982). La durée d'éclairement par nycthémère était de 14 heures.

La diète hydrique commençait vers $17 \mathrm{~h}$., avait une durée totale de $64 \mathrm{~h}$., et se terminait vers $9 \mathrm{~h}$. du matin. Deux prises de sang étaient faites en vue du dosage de corticostérone plasmatique, approximativement entre $14 \mathrm{~h}$. et $15 \mathrm{~h}$., l'une 7 jours avant le début du jeûne, l'autre après un peu moins de $48 \mathrm{~h}$. de jeûne. La première mesure correspondait donc à une manipulation sans mise en œuvre du facteur d'agression étudié, et dans des conditions contrôlables d'environnement identiques à celles de la seconde mesure. La température rectale était prise par une sonde reliée à un thermocouple, entre $9 \mathrm{~h}$. et $10 \mathrm{~h}$. du matin, l'un des jours précédents la période de jeûne, puis à la fin de celui-ci.

Deux pesées (avec correction si un œuf était pondu le jour de la mesure) étaient faites, également entre $9 \mathrm{~h}$. et $10 \mathrm{~h}$. le matin, l'une la veille de la mise à jeûn, l'autre en fin de jeûne, en même temps que la mesure de température. Une nouvelle pesée avait lieu 4 semaines après ces dernières mesures. Les variations de poids sont exprimées en valeur absolue et en $\%$ du poids corporel de départ dans chaque cas. Enfin, le nombre d'œufs pondus a été enregistré pour chaque poule dans la période de 4 semaines mentionnée ci-dessus.

La corticostérone dans le plasma sanguin a été déterminée par dosage radioimmunologique à l'aide d'un antisérum anticorticostérone 21 Thyroglobuline. Cet anticorps présente des réactions croisées avec la 11 désoxycorticostérone $(27 \%)$, la progestérone $(17 \%)$, le cortisol $(7,5 \%)$. Les réactions croisées avec les autres stéroïdes sont inférieures à 4,6\% . Chez les volailles, les taux sanguins de cortisol sont faibles comparés à ceux de la corticostérone et l'on n'a pas détecté de quantités importantes de désoxycorticostérone. Ces interférences dans le dosage sont donc peu importantes. En revanche, il est nécessaire d'éliminer la progestérone avant la détermination de la corticostérone. Nous avons donc effectué deux extractions successives du plasma sanguin. La première avec $5 \mathrm{ml}$ d'isooctane pour $1 \mathrm{ml} \mathrm{de}$ 
plasma enlève $90 \%$ de la progestérone et $0,8 \%$ de la corticostérone. La deuxième extraction avec $10 \mathrm{ml}$ de dichlorométhane permet de récupérer $94 \%$ de la corticostérone. Le dosage radioimmunologique est donc effectué sur ce deuxième extrait sans chromatographie préalable.

La méthode de dosage est très voisine de celle utilisée par ETCHES (1976). Nous avons utilisé comme traceur de la corticostérone ${ }^{3} \mathrm{H}$ d'activité spécifique $76 \mathrm{C} / \mathrm{mM}$ et la séparation des fractions libres et liées a été effectuée par le charbon-dextran. La sensibilité de la méthode est de $20 \mathrm{pg}$.

\section{Résultats et discussion}

Les résultats sont consignés dans le tableau 1.

\section{TABLEAU 1}

Paramètres physiologiques de poules naines $(d w)$ et normales $\left(D w^{+}\right)$ en réponse à une privation d'aliment.

Physiological parameters of dwarf (dw) and normal $\left(D w^{+}\right)$hens in response to food deprivation

\begin{tabular}{|c|c|c|c|c|c|}
\hline \multirow{2}{*}{ Variable } & \multicolumn{4}{|c|}{ Valeur moyenne } & \multirow{2}{*}{$\begin{array}{c}\text { Valeur de } \\
\text { et } \\
\text { significatio }\end{array}$} \\
\hline & $\mathrm{Dw}^{+}$ & & $d w$ & & \\
\hline $\begin{array}{l}\text { Corticostérone plasmatique avant } \\
\text { jeûne }(\mathrm{ng} / \mathrm{ml}) \ldots \ldots \ldots \ldots \ldots \ldots \ldots \ldots\end{array}$ & $1,16 \pm$ & $\pm 0,11$ & $2,09=$ & $\pm \quad 0,24$ & $3,28 * * *$ \\
\hline $\begin{array}{l}\text { Corticostérone plasmatique après } \\
\text { jeûne de } 46 \mathrm{~h} .(\mathrm{ng} / \mathrm{ml}) \ldots \ldots \ldots \ldots \ldots\end{array}$ & $1,49 \pm$ & $\pm 0,16$ & $3,43=$ & $\pm \quad 0,38$ & $4,30^{* * *}$ \\
\hline Différence $\ldots \ldots \ldots \ldots \ldots \ldots \ldots$ & $0,33 \pm$ & $\pm 0,16$ & $1,35=$ & $\pm 0,33$ & $2,57 * *$ \\
\hline Température rectale avant jeûne $\left({ }^{\circ} \mathrm{C}\right)$ & $40,53 \pm$ & $\pm 0,06$ & $40,28=$ & $\pm 0,07$ & $5,10^{* * *}$ \\
\hline $\begin{array}{l}\text { Température rectale en fin de jeûne } \\
\left({ }^{\circ} \mathrm{C}\right) \ldots \ldots \ldots \ldots \ldots \ldots \ldots \ldots \ldots\end{array}$ & $40,28 \pm$ & $\pm 0,07$ & $39,75=$ & $\pm 0,08$ & $5,03^{* * *}$ \\
\hline Différence $\ldots \ldots \ldots \ldots \ldots \ldots \ldots \ldots$ & $0,25 \pm$ & $\pm \quad 0,09$ & $0,53 \pm$ & $\pm \quad 0,08$ & $2,49 *$ \\
\hline Poids corporel initial $(g) \ldots \ldots \ldots$ & $1739,1 \pm$ & $\pm 30,6$ & $1039,2=$ & $\pm 16,9$ & $17,26 * * *$ \\
\hline Perte de poids en fin de jeûne $(\mathrm{g})$.. & $-121,8 \pm$ & $\pm \quad 6,4$ & $-106,1=$ & $\pm \quad 4,4$ & $2,02 *$ \\
\hline $\begin{array}{l}\text { Perte de poids en fin de jeûne, } \% \text { du } \\
\text { poids initial (1) } \ldots \ldots \ldots \ldots \ldots \ldots \ldots\end{array}$ & $-7,0 \pm$ & $\pm \quad 0,4$ & $-10,3=$ & $\pm \quad 0,4$ & $5,85 * * *$ \\
\hline $\begin{array}{l}\text { Reprise de poids dans le mois suivant } \\
\text { la fin du jeûne }(\mathrm{g}) \ldots \ldots \ldots \ldots \ldots \ldots\end{array}$ & $139,0 \pm$ & $\pm 24,5$ & $119,8=$ & $\pm 18,0$ & 0,63 \\
\hline $\begin{array}{l}\text { Reprise de poids, } \% \text { du poids en fin } \\
\text { de jeûne }(1) \ldots \ldots \ldots \ldots \ldots \ldots \ldots\end{array}$ & $9,1 \pm$ & $\pm 1,4$ & $13,3 \pm$ & $\pm \quad 1,7$ & 1,69 \\
\hline $\begin{array}{l}\text { Nombre d'œufs dans le mois suivant } \\
\text { la fin du jeûne } \ldots \ldots \ldots \ldots \ldots \ldots \ldots\end{array}$ & $10,4 \pm$ & $\pm \quad 1,6$ & $6,0=$ & $\pm \quad 1,2$ & $2,20^{*}$ \\
\hline
\end{tabular}


Pour les paramètres envisagés (poids et température corporelle, corticostérone) on retrouve les effets connus d'une diète hydrique (BELL et FREEMAN, 1971) quel que soit le génotype.

L'objet essentiel du présent travail était la comparaison des génotypes. Les poules naines ont déjà un taux de corticostérone plasmatique significativement plus élevé que les poules de taille normale en alimentation ad libitum. La privation d'aliment accentue cette différence. Un résultat antérieur obtenu en septembre 1975 (données non publiées) en alimentation ad libitum seulement et dans une population différente (plus lourde et du type " œuf brun ") ne montrait, par contre, pas d'effet associé au gène $\mathrm{dw}$ : sur 25 couples de sœurs, l'une de génotype $\mathrm{Dw}^{+}$, l'autre dw, âgées de 10 mois et en ponte, le taux moyen $(\mathrm{ng} / \mathrm{ml})$ de corticostérone plasmatique était respectivement de 0,90 et 0,87 . Comme il a déjà été signalé, MAUldin (1978) trouve, chez des jeunes, un effet associé au gène dw différent selon le poids moyen de la lignée considérée. Il paraît donc y avoir une analogie avec nos propres résultats.

La température rectale est significativement inférieure pour les poules dw lorsque l'aliment est distribué ad libitum. Ceci s'accorde avec nos observations antérieures sur un échantillon plus important (BANERJEE et al., 1982) ainsi qu'avec les résultats d'autres auteurs passés en revue par Guillaume (1976). Ce dernier rapproche ces résultats de l'hypothyroïdisme léger des oiseaux nains. De plus, la baisse de température après jeûne est un peu plus grande - d'environ $0,3^{\circ} \mathrm{C}-$ chez les poules $\mathrm{dw}$. La perte de poids en un peu plus de 60 heures de jeûne est relativement peu différente en valeur absolue dans les deux génotypes, mais corrélativement, elle est plus élevée en pourcentage du poids de départ chez les naines. Des trois points de vue du taux de corticostérone, de la température interne et de la perte de poids en pourcentage, la réponse du génotype dw au jeûne apparaît donc plus importante que celle des poules $\mathrm{Dw}^{+}$. Inversement, la reprise de poids des naines dans les quatre semaines qui suivent est la plus importante, en pourcentage du moins. La différence des génotypes en pourcentage n'est pas significative, toutefois la variance résiduelle est beaucoup plus élevée que celle de la perte de poids lors du jeûne. Enfin, le nombre d'œufs pondus durant ces quatre semaines est faible pour les deux génotypes, mais particulièrement pour les poules naines.

Les corrélations phénotypiques entre les variables ci-dessus, regroupées sur une base intra-génotype, ne sont pas significatives pour la plupart, et nous n'en donnons pas le détail. En particulier, on ne peut mettre en évidence de corrélations entre les taux hormonaux d'une part, les variations de poids corporel ou celles de température avant et après jeûne de l'autre. Notons cependant une corrélation négative $(\mathrm{r}=-0,36 ; \mathrm{P}<0,01)$ entre le poids corporel en début d'expérience et le taux de corticostérone après jeûne. La corrélation du poids corporel avec le taux de corticostérone avant jeûne est de même sens $(r=-0,25)$ mais non significative. En d'autres termes, les poules de petite taille présentent un taux de corticostérone plus élevé, surtout pendant une diète hydrique : le parallélisme avec l'effet du gène $d w$ suggère que cet effet peut être une conséquence secondaire de la réduction de taille.

La privation temporaire d'aliment est supposée exclue en élevage industriel moderne. Elle peut cependant se rencontrer dans les conditions plus difficiles de pays en développement. Nos résultats indiquent que des pondeuses naines pourraient y être plus sensibles que les poules de taille normale. 


\section{Références bibliographiques}

Banerjee A.K., Bordas A., Merat P., 1982. Sex-linked dwarf gene (dw) in White Leghorn laying hens under normal and hot temperature. Ann. Génét. Sél. anim., 14, 3-27.

Bell D.J., Freeman B.M., 1971. Physiology and biochemistry of the domestic fowl. Academic Press, London.

ETCHES R.J., 1976. A radioimmunoassay for corticosterone and its application to the measurement of stress in poultry. Steroids, 28, 763-773.

FreEMAN B.M., 1976. Stress and domestic fowl : A physiological reappraisal. World's Poult. Sci. J., 32, $249-256$.

Gross W.B., Siegel P.B., 1973. Effects of social stress and steroids on antibody production. Avian Dis., 17, 807-815.

Gross W.B., Siegel P.B., 1981. Long-term exposure of chicks to three levels of social stress. Avian Dis., 25, 312-325.

Guillaume J., 1976. The dwarf gene $\mathrm{dw}$ : its effects on anatomy, physiology, nutrition, management. Its application in poultry industry. World's Poult. Sci. J., 32, 285-301.

MAUldin J.M., 1978. Genetics, behaviour and disease resistance in chickens. Ph. D. Thesis, Virginia State University, U.S.A.

Mauldin J.M., Siegel P.B., Gross W.B., 1978. Dwarfism in diverse genetic backgrounds. 2. Behaviour and disease resistance. Poult. Sci., 57, 1488-1492. 